\title{
Schwarzschild black hole can also produce super-radiation phenomena
}

\author{
Wen-Xiang Chen* \\ Department of Astronomy, \\ School of Physics and Materials Science, \\ GuangZhou University
}

\begin{abstract}
According to traditional theory, the Schwarzschild black hole does not produce super radiation. If the boundary conditions are set in advance, the possibility is combined with the wave function of the coupling of the boson in the Schwarzschild black hole, and the mass of the incident boson acts as a mirror, so even if the Schwarzschild black hole can also produce super-radiation phenomena.
\end{abstract}

Keywords: Schwarzschild black hole, superradiance, Wronskian determinant

\section{INTRODUCTION}

In a closely related study in 1962, Roger Penrose proposed a theory that by using point particles, it is possible to extract rotational energy from black holes. The Penrose process describes the fact that the Kerr black hole energy layer region may have negative energy relative to the observer outside the horizon. When a particle falls into the energy layer region, such a process may occur: the particle from the black hole To escape, its energy is greater than the initial state. It can also show that in Reissner-Nordstrom (charged, static) and rotating black holes, a generalized ergoregion and similar energy extraction process is possible.

The Penrose process is a process inferred by Roger Penrose that can extract energy from a rotating black hole. Because the rotating energy is at the position of the black hole, not in the event horizon, but in the area called the energy layer in Kerr space-time, where the particles must be like a propelling locomotive, rotating with space-time, so it is possible to extract energy . All objects in the energy horizon are dragged by rotating time and space. In this process, a mass of matter enters the energy horizon of the black hole, and once it enters the energy horizon, it will be split into two groups. The momentum of these two groups is reorganized, so one of them will escape to infinity, while the other will fall through the event horizon and fall into the black hole. The escaping material fragments may carry more mass energy than the originally entered mass, while the fragments entering the black hole carry negative mass energy. In summary, this process reduces the angular momentum of the black hole and reduces the corresponding energy conversion, because the lost momentum is bound to be extracted by energy. Unlike the Penrose process, superradiation mainly involves incident waves and outgoing waves. It is a quantum process.

We know that the fluctuation simulation of the Penrose process is called superradiation. When describing superradiation, what we are discussing about incident matter is not a massive object such as a spacecraft, but a wave packet flying in the direction of a black hole. The wave packet propagates as described by the corresponding wave equation (we will see such a wave equation in more detail later). As a result, part of the wave will "fall into" the black

\footnotetext{
*Electronic address: wxchen4277@qq.com
} 
hole, while the rest will pass through the black hole and eventually leave the black hole region. If the energy of the outgoing wave is greater than the energy of the oncoming incident wave, then people will say that it is superradiative scattering. This effect is very similar to the Penrose process. However, one main difference is that, compared with the Penrose process, the momentum of the emitted wave is not arbitrary. On the contrary, the state of the emitted wave is completely determined by the wave equation, and the only thing that is not determined is the one that is ready to be incident. Wave packet. According to the black hole bomb mechanism proposed by Press and Teukolsky, if there is a mirror between the black hole horizon and the black hole to prevent the scattered waves from being scattered to infinity, the amplified waves can be reflected back and forth between the mirror and the black hole and grow exponentially. This makes the superradiation of the black hole unstable.

The Kerr-black hole-scalar field mirror system first designed by Press and Teukolsky has attracted the attention of physicists in the past four decades. From the literature on the numerical study of this kind of rotating black hole bomb, we know an interesting fact, that is, if the reflector is too close to the black hole horizon, super radiation will produce a stabilizing effect. And we know that black holes need to meet the conditions to produce classical superradiation instability:

(1) The incident perturbation field is the Bose field;

(2) The black hole is rotating or charged;

(3) Meet the super-radiation conditions $0<\omega<m \omega_{H}$;

(4) The existence of reflective mirrors.

Among them (1), (2), (3) are the conditions for generating super radiation. In 1972, Press and Teukolsky[15] proposed that It is possible to add a mirror to the outside of a black hole to make a black hole bomb (according to the current explanation, this is a scattering process involving classical mechanics and quantum mechanics[1, 2, $9,10,13,14,16])$.Regge and Wheeler proved that the spherically symmetric Schwarzschild black hole is stable under disturbance. Due to the significant influence of super radiation, the stability of rotating black holes is more complicated. Superradiation effects can occur in classical and quantum scattering processes. When a boson wave hits a rotating black hole, if certain conditions are met, the black hole may be as stable as a Schwarzschild black hole. When a boson wave hits a rotating black hole, if the frequency range of the wave is under superradiation conditions, the wave reflected by the event horizon will be amplified.

This means that when the incident wave is scattered, the wave extracts rotational energy from the rotating black hole. According to the black hole bomb mechanism proposed by Press and Teukolsky, if there is a mirror between the black hole horizon and the outer space, the magnified wave can be reflected back and forth between the mirror and the black hole and grow exponentially. This leads to the superradiation instability of black holes. Many authors have studied the black hole stability of the superradiation mechanism. In the universe, the real black hole does not exist in isolation, but constantly interacts with the surrounding matter. Therefore, in the fields of black hole physics and astrophysics, the study of the external disturbance field of black holes has always been a subject of much concern. By analyzing the disturbance field outside the black hole, the stability of the black hole can be judged. When a black hole is subjected to a small disturbance, if the disturbance field weakens in the later stage of evolution, such a black hole is stable. Conversely, if the perturbation field rises in the later stage of evolution, the black hole is unstable. Such black holes will disappear or become other objects. Recently, the Kerr black hole has been shown to be stable to some mass scalar disturbances. 
When a bosonic wave is impinging upon a rotating black hole, the wave reflected by the event horizon will be amplified if the wave frequency $\omega$ lies in the following superradiant regime[11, 12, 15, 17, 18]

$$
0<\omega<m \Omega_{H}, \Omega_{H}=\frac{a}{r_{+}^{2}+a^{2}}
$$

where $m$ is azimuthal number of the bosonic wave mode, $\Omega_{H}$ is the angular velocity of black hole horizon. This amplification is superradiant scattering. Therefore, through the superradiation process, the rotational energy of the black hole can be extracted. If there is a mirror between the black hole's horizon and infinite space, the amplified wave will scatter back and forth and grow exponentially, which will cause the black hole's superradiation to become unstable.

Associate Professor Hasegawa Yuji of the Vienna University of Technology and Professor Masaaki Ozawa of Nagoya University and other scholars published empirical results against Heisenberg's uncertainty principle on January 15, 2012[8]. They used two instruments to measure the spin angle of neutrons and calculated them, and they got a measurement result with a smaller error than the Heisenberg uncertainty principle, which proved the measurement advocated by the Heisenberg uncertainty principle. The limit is wrong. However, the uncertainty principle is still correct, because this is the inherent quantum nature of the particle.

In the article[6] follows the method I used to study superradiation and connects the uncertainty principle with the superradiation effect. I found that under the superradiation effect, the measurement limit of the uncertainty principle can be smaller.From that article, we can know that if the boundary conditions are not preset, then for the incident interference of the black hole and the coupling wave function of the black hole, the probability flow density equation is equal on both sides. However, if the boundary conditions of the incident boson are set in advance, then the two sides of the probability flow density equation are not equal, because setting the boundary conditions implies a certain probability. This also explains and explains why the no-hair theorem is not valid under the quantum effect(the boundary condition can be $\mu=y \omega)[3][4][5][7]$. According to the traditional theory, the Schwarzschild black hole does not produce superradiation. And if the boundary conditions are preset, the probability of generation is combined with the wave function of the boson coupling in the Schwarzschild black hole, and the mass of the incident boson acts as a reflecting mirror, then even the Schwarzschild black hole can produce superradiation phenomenon.

\section{DESCRIPTION OF THE SCHWARZSCHILD-BLACK-HOLE SYSTEM}

The metric of the Schwarzschild black hole[11, 12] (in natural unit $\mathrm{G}=\mathrm{c}=1$ ) is(contrast the metric of KERR black hole)

$$
\begin{gathered}
d s^{2}=\frac{\Delta}{\rho^{2}} d t^{2}-\frac{\rho^{2}}{\Delta} d r^{2}-\rho^{2} d \theta^{2}-\frac{\sin ^{2} \theta}{\rho^{2}}\left[\left(r^{2}\right) d \phi\right]^{2} \\
\Delta=r^{2}-2 M r, \rho^{2}=r^{2}
\end{gathered}
$$

We know the Klein-Gordon equation

$$
\left(\nabla^{\nu} \nabla_{\nu}-\mu^{2}\right) \Psi=0
$$


Eigenvalues of the above formula and spherical harmonic functions can be written as

$$
\Psi_{\operatorname{lm}}(\mathrm{t}, \mathrm{r}, \theta, \phi)=\sum_{\mathrm{l}, \mathrm{m}} \mathrm{e}^{\mathrm{im} \phi} \mathrm{S}_{\operatorname{lm}}(\theta) \mathrm{R}_{\operatorname{lm}}(\mathrm{r}) \mathrm{e}^{-\mathrm{i} \omega \mathrm{t}}
$$

\section{THE SUPERRADIATION EFFECT OF BOSON SCATTERING}

The Klein-Gordon equation can be written that

$$
\Phi_{; \mu}^{; \mu}=0
$$

where $\Phi_{; \mu} \equiv\left(\partial_{\mu}-i e A_{\mu}\right) \Phi$ and $e$ is the charge of the scalar field. We get $A^{\mu}=\left\{A_{0}(x), 0\right\}$, and $e A_{0}(x)$ can be equal to $\mu($ where $\mu$ is the mass).

$$
A_{0} \rightarrow \begin{cases}0 & \text { as } x \rightarrow-\infty \\ V & \text { as } x \rightarrow+\infty\end{cases}
$$

We know that $\Phi=e^{-i \omega t} f(x)$, and the ordinary differential equation

$$
\frac{d^{2} f}{d x^{2}}+\left(\omega-e A_{0}\right)^{2} f=0
$$

We see that particles coming from $-\infty$ and scattering off the potential with reflection and transmission amplitudes $\mathcal{R}$ and $\mathcal{T}$ respectively. With these boundary conditions, the solution to behaves asymptotically as

$$
\begin{gathered}
f_{\text {in }}(x)=\mathcal{I} e^{i \omega x}+\mathcal{R} e^{-i \omega x}, x \rightarrow-\infty, \\
f_{\text {in }}(x)=\mathcal{T} e^{i k x}, x \rightarrow+\infty
\end{gathered}
$$

where $k= \pm(\omega-e V)$.

The reflection coefficient and transmission coefficient depend on the specific shape of the potential $A_{0}$. We show that the Wronskian

$$
W=\tilde{f}_{1} \frac{d \tilde{f}_{2}}{d x}-\tilde{f}_{2} \frac{d \tilde{f}_{1}}{d x},
$$

between two independent solutions, $\tilde{f}_{1}$ and $\tilde{f}_{2}$, of is conserved. From the equation on the other hand, if $f$ is a solution then its complex conjugate $f^{*}$ is another linearly independent solution. We find $|\mathcal{R}|^{2}=|\mathcal{I}|^{2}-\frac{\omega-e V}{\omega}|\mathcal{T}|^{2}$.Thus,for $0<\omega<e V$, it is possible to have superradiant amplification of the reflected current, i.e, $|\mathcal{R}|>|\mathcal{I}|$. There are other potentials that can be completely resolved, which can also show superradiation explicitly.

We can pre-set the boundary conditions $e A_{0}(x)=y \omega$ (which can be $\left.\mu=y \omega\right)[3][4][5][7]$, and we see that when $y$ is relatively large(according to the properties of the boson, $y$ can be very large), $|\mathcal{R}|^{2} \geq-\frac{\omega-e V}{\omega}|\mathcal{T}|^{2}$ may not hold.In the end,we can get $\Delta x \Delta p \geq 1 / 2$ may not hold.If the boundary conditions of the incident boson are set in advance, the two sides of the probability flow density equation are not equal, because setting the boundary conditions implies a certain probability, which also explains why the no-hair theorem is not valid in quantum effects. 


\section{SCHWARZSCHILD BLACK HOLE CAN ALSO PRODUCE SUPER-RADIATION PHENOMENA}

According to traditional theory, the Schwarzschild black hole does not produce super radiation. If the boundary conditions are set in advance, the possibility is combined with the wave function of the coupling of the boson in the Schwarzschild black hole, and the mass of the incident boson acts as a mirror, so even if the Schwarzschild black hole can also produce super-radiation phenomena.

\section{SUMMARY}

This article proposes a new possibility that Schwarzschild black hole can produce superradiation phenomena, which implies that no matter it is RN black hole or other black holes, the no-hair theorem may not hold under quantum effects.

[1] Berti, E., Cardoso, V. and Casals, M. [2006], 'Eigenvalues and eigenfunctions of spin-weighted spheroidal harmonics in four and higher dimensions', Physical Review D 73(2), 024013.

[2] Cardoso, V., Dias, O. J., Lemos, J. P. and Yoshida, S. [2004], 'Black-hole bomb and superradiant instabilities', Physical Review D 70(4), 044039.

[3] Chen, W.-X. [2020a], 'Chronology protection conjecture may not hold', SSRN Electronic Journal .

URL: https://dx.doi.org/10.2139/ssrn.3645919

[4] Chen, W.-X. [2020b], 'The possibility of the no-hair theorem being violated', Available at SSRN 3569639.

URL: https://dx.doi.org/10.2139/ssrn.3569639

[5] Chen, W.-X. [2020c], 'The strong cosmic censorship conjecture may be violated', SSRN Electronic Journal .

URL: https://dx.doi.org/10.2139/ssrn.3610419

[6] Chen, W.-X. [2020d], 'Uncertainty principle and super-radiance', SSRN Electronic Journal .

URL: https://dx.doi.org/10.2139/ssrn.3683008

[7] Chen, W.-X. and Huang, Z.-Y. [2019], 'Superradiant stability of the kerr black hole', International Journal of Modern Physics D

URL: https://doi.org/10.1142/S0218271820500091

[8] Erhart, Jacqueline; stephan Sponar, G. S. G. B. M. O. Y. H. [2012], 'Experimental demonstration of a universally valid error-disturbance uncertainty relation in spin-measurements', Nature Physics .

[9] Greiner, W., Müller, B. and Rafelski, J. [1985], Evolution of the vacuum state in supercritical potentials, in 'Quantum Electrodynamics of Strong Fields', Springer, pp. 257-299.

[10] Hartman, T., Song, W. and Strominger, A. [2010], 'Holographic derivation of kerr-newman scattering amplitudes for general charge and spin', Journal of High Energy Physics 2010(3), 118.

[11] Herdeiro, C. A. and Radu, E. [2014], 'Kerr black holes with scalar hair', Physical review letters 112(22), 221101.

[12] Kerr, R. P. [1963], 'Gravitational field of a spinning mass as an example of algebraically special metrics', Physical review letters 11(5), 237.

[13] Manogue, C. A. [1988], 'The klein paradox and superradiance', Annals of Physics 181(2), 261-283.

[14] Penrose, R. [2002], 'Gravitational collapse: The role of general relativity (reprinted from rivista del nuovo cimento, numero speziale, i, vol 257, 1969)', General Relativity and Gravitation 34(7), 1141-1165. 
[15] Press, W. H. and Teukolsky, S. A. [1973], 'Perturbations of a rotating black hole. ii. dynamical stability of the kerr metric', The Astrophysical Journal 185, 649-674.

[16] Teukolsky, S. A. [1973], 'Perturbations of a rotating black hole. 1. fundamental equations for gravitational electromagnetic and neutrino field perturbations', Astrophys. J. 185, 635-647.

[17] Vilenkin, A. [1978], 'Exponential amplification of waves in the gravitational field of ultrarelativistic rotating body', Physics Letters B 78(2-3), 301-303.

[18] Zel'dovich, Y. B. [1972], 'Pis' ma zh. eksp. teor. fiz. 14, 270 (1971)[jetp lett. 14, 180 (1971)]', Zh. Eksp. Teor. Fiz 62, 2076. 